Check for updates

Cite this: RSC Adv., 2017, 7, 27189

Received 25th April 2017

Accepted 16th May 2017

DOI: 10.1039/c7ra04611a

rsc.li/rsc-advances

\section{High-efficiency perovskite solar cells employing a conjugated donor-acceptor co-polymer as a hole-transporting material $\dagger$}

Ze Yu, ${ }^{\text {a }}$ Yuchen Zhang, ${ }^{a}$ Xiaoqing Jiang, ${ }^{a}$ Xiaoxin Li, ${ }^{a}$ Jianbo Lai, ${ }^{a}$ Maowei Hu, ${ }^{a}$ Mohammed Elawad, ${ }^{a}$ Gagik G. Gurzadyan, ${ }^{a}$ Xichuan Yang (D) a and Licheng Sun (DD *ab

In this work, we have successfully introduced 2,3,5,6-tetrafluoro-7,7,8,8-tetracyanoquinodimethane (F4TCNQ) as an efficient p-type dopant for donor-acceptor (D-A) co-polymer poly[2,6-(4,4-bis-(2ethylhexyl)-4H-cyclopenta[2,1-b;3,4- $b^{\prime}$ ]dithiophene)-alt-4,7(2,1,3-benzothiadiazole)] (PCPDTBT) as an HTM in mesoscopic perovskite solar cells (PSCs). The bulk conductivity is significantly enhanced by 4 orders of magnitude when PCPDTBT is doped with F4TCNQ $(6 \%, w / w)$. UV-vis and Fourier transform infrared spectroscopy (FTIR) results indicate the occurrence of $p$-doping, which results in higher bulk conductivity. The high conductivity leads to an impressive overall efficiency of $15.1 \%$, which is considerably higher than the pristine PCPDTBT based devices (9.2\%). The superior performance obtained should be largely attributed to the significant enhancement of the photocurrent density strongly correlated with a more efficient charge collection. This is the highest efficiency reported so far for PCPDTBT-based PSCs. Thus, molecularly $p$-doping has been demonstrated to be an effective strategy for further improving the performance of a wide range of D-A and other types of polymeric HTMs in PSCs.

\section{Introduction}

Renewable energy sources have captured worldwide attention with an aim to resolve the issues of rapidly growing energy demands and environmental pollution caused by the combustions of fossil fuels. ${ }^{1}$ Photovoltaic devices that convert solar energy directly to electrical energy represent promising renewable alternatives to fossil fuels. Recently, inorganic-organic hybrid metal halide perovskites with the composition $\mathrm{ABX}_{3}[\mathrm{~A}=$ $\mathrm{CH}_{3} \mathrm{NH}_{3}{ }^{+}$(MA), $\mathrm{NH}=\mathrm{CHNH}_{3}{ }^{+}$(FA) or $\mathrm{Cs}^{+}$; $\mathrm{B}=\mathrm{Pb}$ or $\mathrm{Sn} ; \mathrm{X}=\mathrm{I}$, $\mathrm{Br}, \mathrm{Cl}$ ] have attracted considerable research attention as light absorbers in solid-state thin film solar cells, due to some of their outstanding properties, such as a broad range of light absorption, high extinction coefficient, ambipolar charge transport, high charge mobility and long electron-hole diffusion lengths etc. $^{2-5}$ The power conversion efficiency (PCE) of perovskite solar cells (PSCs) has been rapidly increased to a certified value of $22.1 \%$ in only a few years. ${ }^{6}$ Besides, the fabrication procedure of perovskite is quite simple together with a low production cost.

${ }^{a}$ State Key Laboratory of Fine Chemicals, Institute of Artificial Photosynthesis, DUT-KTH Joint Education and Research Center on Molecular Devices, Dalian University of Technology (DUT), Dalian 116024, China. E-mail: ze.yu@dlut.edu.cn; lichengs@kth.se

${ }^{b}$ Department of Chemistry, School of Chemical Science and Engineering, KTH Royal Institute of Technology, 10044 Stockholm, Sweden

$\dagger$ Electronic supplementary information (ESI) available. See DOI: 10.1039/c7ra04611a
Therefore, PSCs have been considered to be one of the most promising alternatives among the low-cost next-generation solar cell technologies.

High-efficiency PSCs with a typical cell configuration (n-i-p) routinely incorporated hole-transporting materials (HTMs) as ptype contacts between the perovskite film and the cathode material. HTMs play a key role in extracting and collecting the photo-generated holes from the perovskite absorber, thus circumventing undesired recombination losses at the interfaces and enhancing the overall photovoltaic performance. ${ }^{7}$ Therefore, a great deal of efforts has been devoted to exploring effective HTMs for PSCs. A vast number of triphenylamine (TPA)-based organic small molecular HTMs have been developed and tested as HTMs in PSCs. ${ }^{8}$ 2, $2^{\prime}, 7,7^{\prime}$-tetrakis( $N, N$-di- $p$ methoxyphenylamine)-9, $9^{\prime}$-spirobifluorene (spiro-OMeTAD) and $\mathrm{N} 2, \mathrm{~N} 2, \mathrm{~N}^{\prime}{ }^{\prime}, \mathrm{N} 2^{\prime}, \mathrm{N} 7, \mathrm{~N} 7, \mathrm{~N}^{\prime}{ }^{\prime}, \mathrm{N}^{\prime}$-octakis(4-methoxyphenyl)

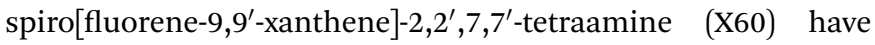
been the most successful examples for these TPA-based small molecular HTMs, exhibiting excellent PCEs of $21.8 \%$ and 19.84\%, respectively. ${ }^{9,10}$ Besides, inorganic p-type semiconductor, such as copper iodide (CuI) and copper thiocyanate (CuSCN) have also attracted attention as HTMs because of their high conductivity, low production cost and simple processing procedures. ${ }^{\mathbf{1 1 - 1 3}}$

Conjugated conducting polymers have also been intensively studied as HTMs in PSCs, owing to their excellent charge carrier mobility, suitable energy levels and good processability. A number of polymeric HTMs have been applied into PSCs, 
including poly(triarylamine) (PTAA), polyfluorene derivatives, polyaniline (PANI), poly(3-hexylthiophene-2,5-diyl) (P3HT) and several thiophene-based conducting polymers. ${ }^{14-21}$ Yet, the overall performance of PSC devices based on these polymeric HTMs were not satisfactory (typically below 15\%). Poly(triarylamine) (PTAA) has been the only example of conducting polymer-based HTMs used in PSCs that could work as efficiently as TPA-type small molecules with the highest reported PCE of more than $20 \% .^{22}$

Donor-acceptor (D-A) co-polymers that possess strong interchain interactions and substantial $\pi$-conjugated morphology, have shown excellent performance as HTMs in PSCs without using any additives. ${ }^{23,24}$ Poly[2,6-(4,4-bis-(2-ethylhexyl)- $4 H^{-}$ cyclopenta[2,1- $\left.b ; 3,4-b^{\prime}\right]$ dithiophene)-alt-4,7(2,1,3-benzothiadiazole)] (PCPDTBT) (Fig. 1a), was a well-known D-A copolymer and has been widely studied as electron donors in organic photovoltaic devices. ${ }^{25-27}$ Owing to its suitable energy levels, easy processability and hydrophobic property, PCPDTBT has also been examined as hole-conductors in PSCs. However, the overall performance of PSCs based on PCPDTBT as HTMs were not satisfactory up till now. Seok et al. first introduced PCPDTBT as a HTM in PSCs together with additives lithium bis(trifluoromethanesulfonyl)imide salt (LiTFSI) and 4-tert-butylpyridine (TBP), obtaining an efficiency of only $5.3 \% .^{20}$ Then, Palomares and co-workers further demonstrated that dopantfree PCPDTBT together with $\mathrm{MAPbI}_{3-x} \mathrm{Cl}_{x}$ light absorber yielded low efficiency of $\sim 5.3 \%$ as well..$^{28}$ Meredith et al. examined an ultrathin $(<10 \mathrm{~nm})$ PCPDTBT p-type transport layer in inverted structural PSCs $(\mathrm{p}-\mathrm{i}-\mathrm{n})$, an average PCE of $7.8 \%$ was obtained that is the maximum efficiency reported so far for PCPDTBT-based PSCs. ${ }^{29}$

Tetrafluoro-tetracyano-quinodimethane (F4TCNQ) (Fig. 1a) has been successfully demonstrated to be an effective molecular p-dopant for conducting polymers, such as P3HT, PTAA and D-A copolymers. ${ }^{30-34}$ Fortunately, the lowest unoccupied molecular orbital (LUMO) energy level of F4TCNQ $(-5.24 \mathrm{eV})$ matches well the highest occupied molecular orbital (HOMO) energy level of PCPDTBT $(-5.30 \mathrm{eV})$ (Fig. 1b), which could facilitate p-type doping via electrons transfer from the HOMO level of the PCPDTBT to the LUMO level of the F4TCNQ. Therefore, more positive charges are expected to be formed in the mixture, thus improving the bulk conductivity of the PCPDTBT film. In this work, we systematically studied the influence of F4TCNQ doped PCPDTBT composites as HTMs on the photovoltaic performance in mesoscopic PSCs. UV-vis and Fourier transform infrared spectroscopy (FTIR) measurements both indicated that p-doping occurred. Under an optimal doping condition $(6 \%, w / w)$, the conductivity of the doped film was increased by 4 orders of magnitude as compared to the pristine one. Owing to the significant increase of the conductivity, the overall efficiency was improved by more than $50 \%$, reaching a best PCE of $15.1 \%$ measured at standard one sun irradiation (100 mW cm ${ }^{-2}$, AM 1.5G). Such high efficiency obtained for F4TCNQ doped PCPDTBT should be attributed to the dramatic enhancements of the short-circuit current density $\left(J_{\mathrm{sc}}\right)$ and fill factor $(\mathrm{FF})$ originated from the high conductivity. This value is the highest efficiency reported thus far for PSCs based PCPDTBT as HTMs. Furthermore, PCPDTBT:F4TCNQ composite HTM based PSCs also exhibited excellent long-term stability under ambient atmosphere. The present finding opens up a new avenue for the optimization of D-A copolymerbased HTMs for highly efficient and stable PSCs.

\section{Experimental section}

\subsection{Materials}

All the chemicals and reagents were used as received from chemical companies, including $\mathrm{PbI}_{2}\left(>98 \%\right.$, TCI), $\mathrm{PbBr}_{2}$ (99\%, SigmaAldrich), $\mathrm{HI}$ ( $48 \%$ in water, Sigma-Aldrich), $\mathrm{HBr}(48 \%$ in water, Sigma-Aldrich), $\mathrm{CH}_{3} \mathrm{NH}_{2}$ (33 wt\% in absolute ethanol, SigmaAldrich), formamidine acetate (99\%, Sigma-Aldrich), titanium diisopropoxide bis(acetylactonate) $75 \%$ in isopropanol (Tiacac, Sigma-Aldrich), mesoporous-TiO ${ }_{2}$ paste (18NR-T, Dyesol), 2,3,5,6tetrafluoro-7,7,8,8-tetracyanoquinodimethane (F4TCNQ, 97\%, Sigma-Aldrich), poly[2,6-(4,4-bis-(2-ethylhexyl)-4H-cyclopenta[2,1$\left.b ; 3,4-b^{\prime}\right]$ dithiophene)-alt-4,7(2,1,3-benzothiadiazole)] (PCPDTBT, average $M_{\mathrm{w}} 7000-20000$, Sigma-Aldrich).

The $\mathrm{NH}=\mathrm{CHNH}_{3} \mathrm{I}$ (FAI) and $\mathrm{CH}_{3} \mathrm{NH}_{3} \mathrm{Br}$ (MABr) were synthesized by the same methods according to a previously literature..$^{35}$ The mixed-ion perovskite precursor solution (1.35 $\mathrm{M})$ of $\left(\mathrm{FAPbI}_{3}\right)_{1-x}\left(\mathrm{MAPbBr}_{3}\right)_{x}(x=0.15)$ was prepared in a glovebox, by dissolving the FAI (1 M), MABr $(0.2 \mathrm{M}), \mathrm{PbI}_{2}(1.1 \mathrm{M})$ and $\mathrm{PbBr}_{2}(0.2 \mathrm{M})$ in a mixed solvent of dimethyl formamide (DMF) and dimethyl sulfoxide (DMSO) $(4: 1, \mathrm{v} / \mathrm{v})$, as reported previously. ${ }^{15,36}$

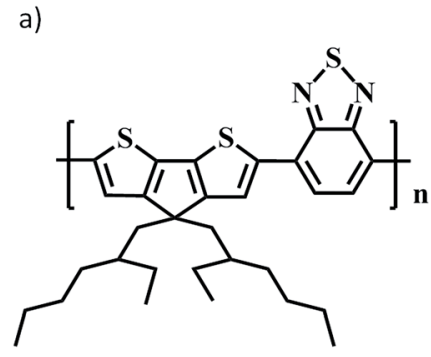

PCPDTBT

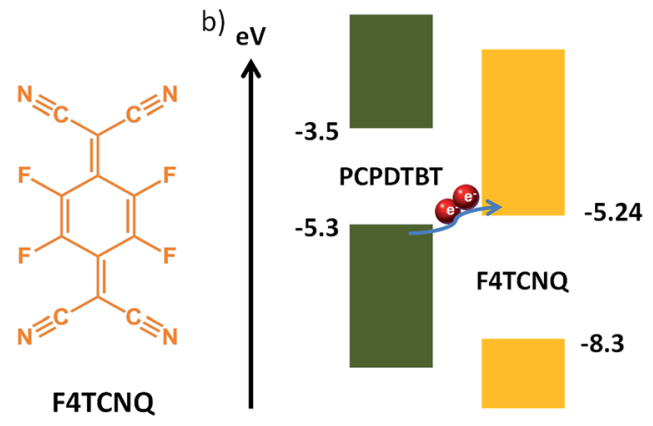

$-8.3$

Fig. 1 (a) Chemical structure of the D-A co-polymer PCPDTBT and F4TCNQ. (b) Energy level diagram of PCPDTBT and F4TCNQ. 


\subsection{Solar cells fabrication and characterization}

The PSC devices were fabricated as described previously. ${ }^{37}$ Fluorine-doped tin oxide (FTO)-coated glass (Pilkington TEC 15) was firstly patterned by etching with $\mathrm{Zn}$ powder and $\mathrm{HCl}(2 \mathrm{M})$. The etched substrate was then sequentially cleaned by using detergent, de-ionized water, acetone and ethanol in ultrasonic bath. Remaining organic residues were removed under oxygen plasma for $30 \mathrm{~min}$. A compact $\mathrm{TiO}_{2}$ blocking layer (BL) of roughly 30-40 $\mathrm{nm}$ was deposited on the cleaned FTO glasses by spray pyrolysis of titanium diisopropoxide bis(acetylacetonate) diluted in anhydrous ethanol at a volumetric ratio of $1: 10$ and then heated at $500{ }^{\circ} \mathrm{C}$ for $30 \mathrm{~min}$. A mesoporous $\mathrm{TiO}_{2}$ layer was deposited by spin-coating $\mathrm{TiO}_{2}$ paste (Dyesol 18NR-T) diluted in anhydrous ethanol at ratio of $1: 5$ by weight at $5000 \mathrm{rpm}$ for $30 \mathrm{~s}$. The layers were then sintered in air at $500{ }^{\circ} \mathrm{C}$ for $30 \mathrm{~min}$. The mixed-ion perovskite films were deposited onto the mesoporous $\mathrm{TiO}_{2} / \mathrm{BL} \mathrm{TiO}_{2} / \mathrm{FTO}$ substrates from the precursor solution by a two-step spin-coating procedure, at $1000 \mathrm{rpm}$ for $10 \mathrm{~s}$ and then $5000 \mathrm{rpm}$ for $30 \mathrm{~s}$. During the second step, $200 \mu \mathrm{L}$ of chlorobenzene was dropped onto the substrates $10 \mathrm{~s}$ prior to the end of the program. The substrates were directly heated on a hotplate at $100{ }^{\circ} \mathrm{C}$ for $60 \mathrm{~min}$. After cooling to room temperature, different doping level of PCPDTBT:F4TCNQ composites HTMs were deposited on the perovskite layers at $2000 \mathrm{rpm}$ for $30 \mathrm{~s}$ via solution process. $20 \mathrm{mg} \mathrm{ml}^{-1}$ PCPDTBT solution was prepared by dissolving $20 \mathrm{mg}$ PCPDTBT in $1 \mathrm{ml}$ ortho-dichlorobenzene (DCB), stirred at $70{ }^{\circ} \mathrm{C}$ for $30 \mathrm{~min}$. P-type doping material F4TCNQ in DCB solution with a concentration of $2 \mathrm{mg}$ $\mathrm{ml}^{-1}$ was also stirred at $70{ }^{\circ} \mathrm{C}$ for $30 \mathrm{~min}$ before adding to the PCPDTBT solution. After spin coating of the HTM layers, the substrates were heated at $65{ }^{\circ} \mathrm{C}$ for $15 \mathrm{~min}$. Finally, a layer of $100 \mathrm{~nm} \mathrm{Au}$ was deposited on top of the HTM layers under high vacuum $\left(<4 \times 10^{-4} \mathrm{~Pa}\right)$ by thermal evaporation.

The photocurrent-voltage $(J-V)$ characteristics of the solar cells were measured using a Keithley 2400 Source-measure unit under illumination of a simulated sunlight (AM 1.5G, $100 \mathrm{~mW}$ $\mathrm{cm}^{-2}$ ) provided by an Oriel Sol3A solar simulator (Newport USA, Model: 94023A) with an AM 1.5 filter in ambient air. Light intensity was calibrated with a Newport calibrated standard $\mathrm{Si}$ reference cell (SER. No: 506/0358). A black mask with a circular aperture $\left(0.09 \mathrm{~cm}^{2}\right)$ smaller than the active area of the square solar cell $\left(0.20 \mathrm{~cm}^{2}\right)$ was applied on top of the cell. The $J-V$ curves were obtained from forward bias to short-circuit at a scan rate of $10 \mathrm{mV} \mathrm{S}^{-1}$. The incident photo-to-current conversion efficiency (IPCE) was obtained by a Hypermono-light (SM-25, Jasco Co. Ltd., Japan). Prior to measurement, a standard silicon solar cell was used as reference.

\subsection{Characterizations}

The UV-vis spectra were obtained by Agilent 8453 spectrophotometer (Model: HP 8435, China). The infrared spectra were measured by Fourier Transform Infrared Spectrometer (FTIR) mode on 6700 (ThermoFisher, USA). The top view and crosssection scanning electron microscopy (SEM) images were obtained by HR-SEM performed with FEI (Field Emission Instruments: Nova Nano SEM 450), the USA. Conductivity measurements were performed as follows. ${ }^{38}$ Glass substrates were sequentially cleaned by detergent, de-ionised water, acetone and ethanol. Remaining organic residues were removed under oxygen plasma for $30 \mathrm{~min}$. A thin layer of compact $\mathrm{TiO}_{2}$ $(\sim 30 \mathrm{~nm})$ was coated on the glass substrates by spray pyrolysis. After sintering the $\mathrm{TiO}_{2}$ film at $500{ }^{\circ} \mathrm{C}$ for $30 \mathrm{~min}$, the film was cooled to room temperature. A solution of HTM in DCB was spin-coated onto the $\mathrm{TiO}_{2}$ substrate, whereas the concentration was the same as in case for the photovoltaic device. Finally, a $200 \mathrm{~nm}$-thick of Ag was deposited on the top of the HTM by thermal evaporation under high vacuum $\left(<4 \times 10^{-4} \mathrm{~Pa}\right)$. A twopoint probe setup was used with a keithley 2400 source meter for measuring linear current-voltage curves. Steady-state photoluminescence (PL) measurements were performed with spectrofluorometer (Horiba Jobin Yvon, Fluorolog-3) at excitation wavelength of $600 \mathrm{~nm}$. Time-resolved photoluminescence was measured by use of time-correlated single photon counting (TCSPC) technique (PicoHarp 300, PicoQuant). For excitation, the second harmonic of femtosecond titanium-sapphire laser (Mai Tai DeepSee, Spectra Physics) at $425 \mathrm{~nm}(150 \mathrm{fs}, 80 \mathrm{MHz})$ was utilized. For TCSPC measurements, the instrument response function is about 40 ps. Deconvolution/fitting procedure was performed by use of commercial software FluoFit Pro (PicoQuant); overall time resolution of the setup is 8-10 ps. All measurements were done at room temperature. The HOMO energy level of the PCPDTBT film was examined using ultraviolet photoelectron spectroscopy (UPS) with photon energy of $40.8 \mathrm{~V}$. A sample bias of $5.0 \mathrm{~V}$ was applied to observe the secondary electron cutoff. The electrochemical impedance spectroscopy (EIS) measurements were carried out at different applied bias in the dark condition using an impedance/gainphase analyser (Zahner Model: Zennium, Serial No. 40037, German) electrochemical workstation with the scanning frequency range from $10^{6}$ to $0.1 \mathrm{~Hz}$. The magnitude of the alternative signal was $10 \mathrm{mV}$.

\section{Results and discussion}

UV-vis absorption spectra of solutions containing PCPDTBT with varied doping concentrations of F4TCNQ are depicted in Fig. 2a and b. For pristine PCPDTBT, there are two absorption peaks due to the $\pi-\pi^{*}$ transition in visible light region. The weaker one belongs to the electron donor part PCPDT at $420 \mathrm{~nm}$, while the other one centred at $740 \mathrm{~nm}$ should be attributed to the strong electron acceptor BT segment. When doped with F4TCNQ with different concentrations (2-8\%, w/w), sub-gap absorption peaks in the infrared region can be distinctly observed as shown in Fig. 2b. The sub-gap absorptions are not seen for both pristine PCPDTBT and F4TCNQ solutions, separately. Such phenomena observed implies the occurrence of ground-state charge transfer from the HOMO level of PCPDTBT to the LUMO level of F4TCNQ, initiated by effective electron removal of electrons due to the electron-poor characteristic of F4TCNQ molecule. ${ }^{30,31}$ A distinct color change in PCPDTBT solution with varied concentrations of F4TCNQ (Fig. 2c) also supports the ground-state charge transfer happening after F4TCNQ doping. This hypothesis was further 
a)
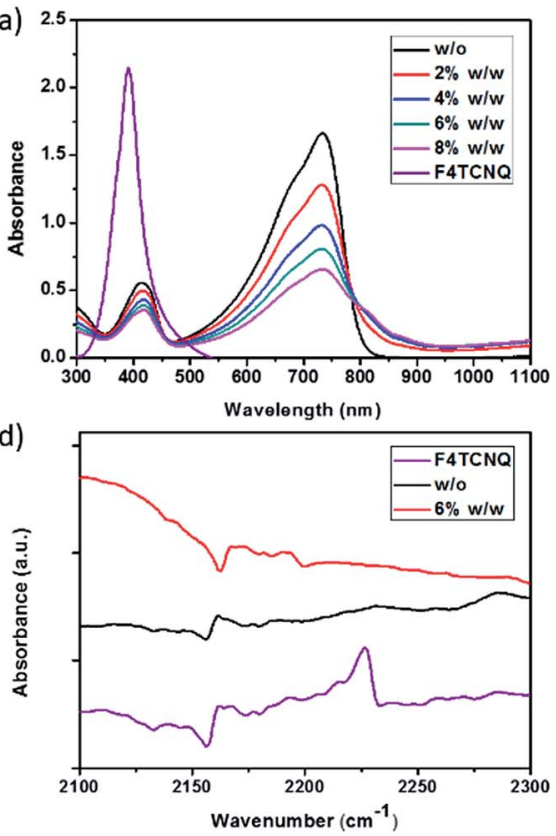

b)

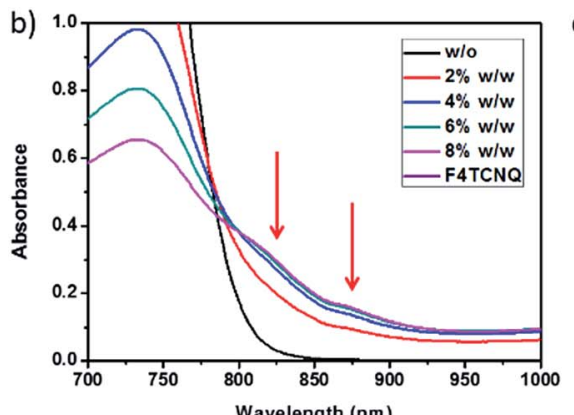

e)
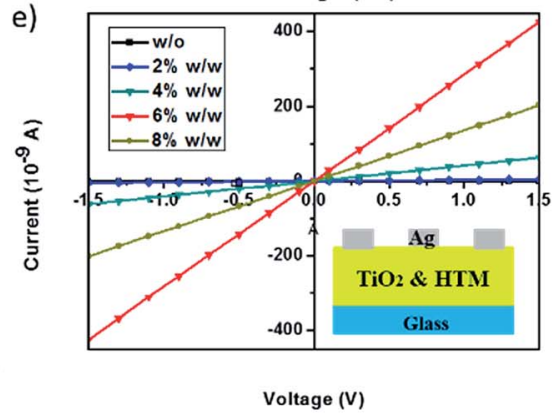

c)

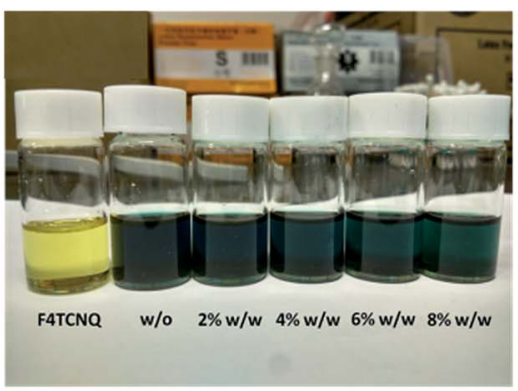

f)

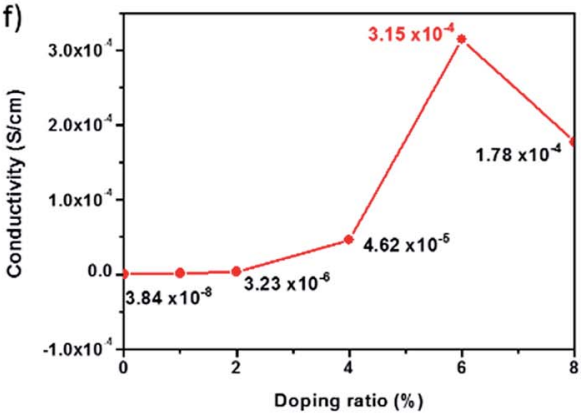

Fig. 2 (a) UV-vis absorption spectra of F4TCNQ solution and PCPDTBT solution doped with different concentrations of F4TCNQ and (b) amplified spectra in the infrared spectral region. (c) Image of different UV-vis measurement solutions. (d) FTIR absorption spectra of F4TCNQ, pristine PCPDTBT and 6\% F4TCNQ doped PCPDTBT films. (e) Current vs. voltage curves of glass/compact TiO $2 / \mathrm{HTM} / \mathrm{Ag}_{\mathrm{g}}$ hole-only devices. (f) Conductivity results of PCPDTBT films doped with different concentrations of F4TCNQ.

confirmed by the FTIR measurement as shown in Fig. 2d. The absorption peak of cyano bond $(\mathrm{C} \equiv \mathrm{N})$ is a sensitive indicator of the presence of charges on F4TCNQ molecules. ${ }^{30}$ The $\mathrm{C} \equiv \mathrm{N}$ absorption peak of neutral F4TCNQ in solid state is centered at $2225 \mathrm{~cm}^{-1}$. However, the $\mathrm{C} \equiv \mathrm{N}$ absorption peak disappears for the F4TCNQ doped PCPDTBT film (6\% taken as an example). These observations indicate the presence of F4TCNQ anion radical state by accepting electrons from PCPDTBT. From the spectroscopic analysis, we can conclude that effective p-type doping occurs when PCPDTBT is mixed with F4TCNQ, which is expected to enhance the bulk conductivity of the PCPDTBT film. Hole conductivity measurements were carried out for PCPDTBT doped with varied concentrations of F4TCNQ using two-point probe methodology based on glass/compact $\mathrm{TiO}_{2} /$ $\mathrm{HTM} / \mathrm{Ag}$ device as reported previously (Fig. 2e).$^{38}$ Conductivity was calculated followed the formula: $\sigma=L /(R \mu d)$, where $L$ is the channel length, $1 \mathrm{~mm} ; R$ is the film resistance calculated from gradients of the curves; $\mu$ is the channel width, $9 \mathrm{~mm} ; d$ is the film thickness, $\sim 100 \mathrm{~nm}$. The conductivity values for different doping ratios are presented in Fig. 2f. Pristine PCPDTBT exhibits a quite low conductivity of $3.84 \times 10^{-8} \mathrm{~S} \mathrm{~cm}^{-1}$. The conductivity values significantly increase with F4TCNQ doping. At an optimal doping level of $6 \% \mathrm{w} / \mathrm{w}$, the conductivity of PCPDTBT film increases by 4 orders of magnitude to $3.15 \times$ $10^{-4} \mathrm{~S} \mathrm{~cm}^{-1}$.

The hole conductivity of a HTM is a very important parameter to guarantee an effective transportation/collection the photo-generated holes from the perovskite absorber. PCPDTBT doped with varied doping concentrations of F4TCNQ were further examined as HTMs in mesoscopic PSCs. The schematic illustration and cross-sectional scanning electron microscopy (SEM) image of the device architecture are depicted in Fig. 3a and $\mathrm{b}$. The solar cell devices were fabricated with a structure of FTO glass/compact $\mathrm{TiO}_{2}(\sim 30-40 \mathrm{~nm}) /$ mesoporous $\mathrm{TiO}_{2}(\sim 150$ $\mathrm{nm}) /$ perovskite/HTM/Au. The mixed-ion perovskite light absorber $\left(\mathrm{FAPbI}_{3}\right)_{0.85}\left(\mathrm{MAPbBr}_{3}\right)_{0.15}$ was prepared by using a solvent-engineering technique as reported previously. ${ }^{15,36}$ Perovskite crystals grew inside the pores of scaffold and additionally formed a capping layer with a total thickness of about $600 \mathrm{~nm}$. SEM top-view image of mixed-ion perovskite film (Fig. S1a†) exhibits dense crystal stacking morphology with an average grain size around $400 \mathrm{~nm}$. PCPDTBT with varied doping levels of F4TCNQ $(0-8 \%, w / w)$ were then spin-coated on top of the perovskite films as HTMs. It can be clearly seen that PCPDTBT:F4TCNQ film uniformly covered onto the compact perovskite layer as shown in Fig. $\mathrm{S} 1 \mathrm{~b} \dagger$ from the SEM top-view image. More device fabrication details can be found in the Experimental section.

The current density-voltage $(J-V)$ characteristics of PSC devices based on PCPDTBT with varied doping levels of F4TCNQ as HTMs measured at $100 \mathrm{~mW} \mathrm{~cm}^{-2}$ illumination (AM 1.5G) are displayed in Fig. S2. $\dagger$ The molecularly p-doping of PCPDTBT with F4TCNQ results in significant improvement of the overall efficiency, owing to the dramatic enhancements of the $J_{\mathrm{sc}}$ strongly correlated to the high conductivity for doped ones. At the doping level of $6 \% \mathrm{w} / \mathrm{w}$, a maximum efficiency was obtained. Therefore, this doping concentration was chosen for the following comparison with the pristine PCPDTBT. As shown in Fig. 2c and Table 1, PSC devices based pristine PCPDTBT show an maximum overall efficiency of only $9.2 \%$, with an open- 
a)
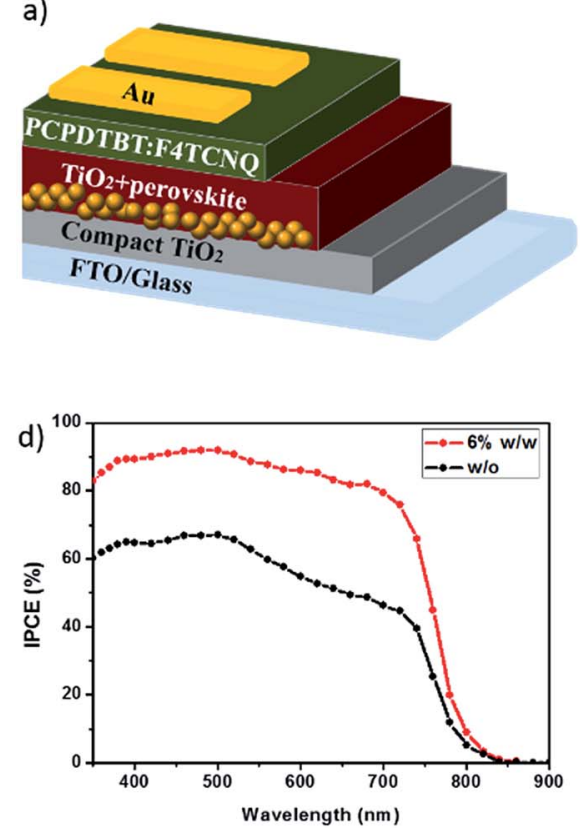
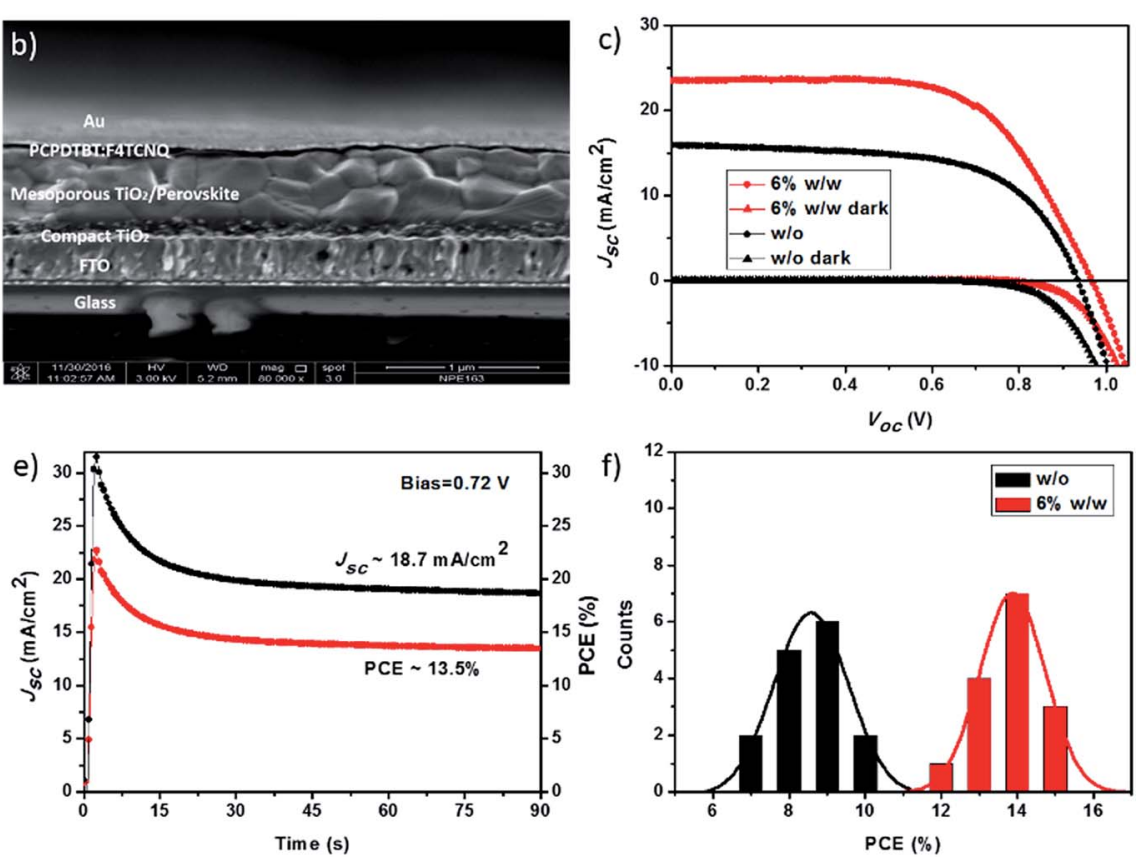

Fig. 3 (a) Schematic device structure of perovskite solar cell. (b) Cross-sectional SEM image of the device consisting of FTO-glass/compact $\mathrm{TiO}_{2} /$ mesoporous $\mathrm{TiO}_{2} /$ perovskite/PCPDTBT:F4TCNQ/Au. (c) $\mathrm{J}-V$ characteristics measured under AM 1.5 simulated sunlight (100 mW $\mathrm{cm}^{-2}$ irradiance) and in the dark. (d) IPCE spectra of PSC devices based on PCPDTBT with and without doping F4TCNQ as HTMs. (e) Steady-state maximum photocurrent (black) and power output (red) measurement for a representative device based on PCPDTBT doped with 6\% F4TCNQ as a HTM. (f) Histogram of power conversion efficiency comparison between PCPDTBT with and without F4TCNQ as HTM (15 cells for each group).

circuit voltage $\left(V_{\text {oc }}\right)$ of $0.93 \mathrm{~V}$, a short-circuit current density $\left(J_{\mathrm{sc}}\right)$ of $15.9 \mathrm{~mA} \mathrm{~cm}^{-2}$ and a fill factor (FF) of 0.62 , respectively. The devices with F4TCNQ doped PCPDTBT exhibits a maximum PCE of $15.1 \%$, with a $V_{\mathrm{oc}}$ of $0.97 \mathrm{~V}$; a $J_{\mathrm{sc}}$ of $23.4 \mathrm{~mA} \mathrm{~cm}^{-2}$ and a FF of 0.67 , respectively. All the photovoltaic parameters are dramatically enhanced after p-doping, in particular for the $J_{\mathrm{sc}}$. This is the highest PCE achieved for PCPDTBT-based HTMs in PSCs up to now.

The incident-photon-to-current conversion efficiency (IPCE) spectra for PSCs based on PCPDTBT with and without F4TCNQ are displayed in Fig. 3d. In general, both of these two devices exhibit a wide absorption extended to over $800 \mathrm{~nm}$. In contrast to pristine PCPDTBT, F4TCNQ doped one presents a remarkable improvement of IPCE over the whole region, yielding a broad IPCE plateau of over $80 \%$ in the range of 350 to $700 \mathrm{~nm}$. It highlights that p-doped PCPDTBT film can effectively transport and collect the photo-generated holes from the perovskite, thus leading to higher charge collection efficiency and photocurrent density.

The $J-V$ hysteresis effects for devices based on PCPDTBT with and without F4TCNQ were evaluated and the results are summarized in Fig. S3 and Table S1 in the ESI. $\dagger$ Devices with F4TCNQ demonstrated a reduced hysteresis as compared to pristine PCPDTBT. The hysteresis indexes are calculated to be 0.50 (without F4TCNQ) and 0.40 (with F4TCNQ), respectively, according to a previous literature. ${ }^{39}$ This result is in good agreement with previous reports, as $J-V$ hysteresis is strongly correlated with the nature of the electronic contact between the perovskite and HTM layer. ${ }^{40-42}$ The enhanced hole conductivity of F4TCNQ doped device may improve hole extraction (reducing the charge accumulation at the interface), thus resulting in a small hysteresis. It was reported that the stabilized power output is a reliable and scan-independent method to determine the efficiency of a PSC device. ${ }^{43}$ The steady-state efficiency of a representative PSC device based on F4TCNQ doped PCPDTBT was measured at a constant bias of $0.72 \mathrm{~V}$ over 90 seconds under

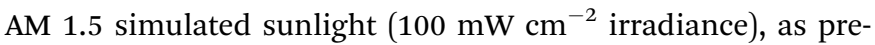
sented in Fig. 3e. The device exhibits a steady-state efficiency of $13.5 \%$ and current density of $18.7 \mathrm{~mA} \mathrm{~cm}^{-2}$ during the testing period. The statistical data of photovoltaic parameters for 15 PSC devices with pristine and doped PCPDTBT as HTMs were also examined. The results are presented and summarized in

Table 1 The photovoltaic parameters of PSC devices using PCPDTBT with (6\% w/w) and without F4TCNQ as HTMs under AM 1.5 simulated sunlight (100 $\mathrm{mW} \mathrm{cm}^{-2}$ irradiance). The data for the best performing cells are concluded in the brackets

\begin{tabular}{|c|c|c|c|c|}
\hline Doping ratio & $V_{\mathrm{oc}}(\mathrm{V})$ & $J_{\mathrm{sc}}\left(\mathrm{mA} \mathrm{cm}^{-2}\right)$ & $\mathrm{FF}$ & PCE (\%) \\
\hline w/o & $0.95 \pm 0.03(0.93)$ & $16.2 \pm 1.3(15.9)$ & $0.60 \pm 0.05(0.62)$ & $9.0 \pm 1.2(9.2)$ \\
\hline $6 \% \mathrm{w} / \mathrm{w}$ & $0.96 \pm 0.02(0.97)$ & $23.1 \pm 1.1(23.4)$ & $0.65 \pm 0.05(0.67)$ & $14.4 \pm 0.7(15.1)$ \\
\hline
\end{tabular}


Fig. 3f and Table 1, respectively. The devices containing F4TCNQ exhibits good reproducibility with an average PCE of $14.4 \pm 0.7 \%$, while devices with pristine PCPDTBT yield an average PCE of only $9.0 \pm 1.2 \%$.

Effective hole extraction from the perovskite absorber is primarily important for a HTM to function well in PSCs. The HOMO energy levels of pristine and F4TCNQ doped PCPDTBT films were measured using ultraviolet photoelectron spectroscopy (UPS). Fig. S4 $\uparrow$ present the cutoff $\left(E_{\text {cutoff }}\right)$ and onset $\left(E_{\mathrm{i}}\right)$ energy regions in the UPS spectrum for pristine and doped PCPDTBT films, respectively. According to the equation $\varphi=$ $40.8-\left(E_{\text {cutoff }}-E_{\mathrm{i}}\right)$, the HOMO energy level of pristine PCPDTBT is calculated to be $-5.3 \mathrm{eV}$, which is in a good agreement with previous results. ${ }^{20}$ No significant difference is found for the F4TCNQ doped PCPDTBT film. The HOMO energy levels of both pristine and doped PCPDTBT films fit well the valence band (VB) of perovskite $\left(\mathrm{FAPbI}_{3}\right)_{0.85}\left(\mathrm{MAPbBr}_{3}\right)_{0.15}(-5.65 \mathrm{eV}) .^{44}$ Thus, an effective extraction of holes from the perovskite can be envisaged. To verify this hypothesis, we carried out steady-state photoluminescence (PL) and time-resolved PL decay characteristics of perovskite/PCPDTBT films. Fig. 4a presents steadystate PL spectra of perovskite film, perovskite with pristine and F4TCNQ doped PCPDTBT, respectively. The perovskite PL emission peak is centred at $775 \mathrm{~nm}$. Strong quenching was observed in the presence of both PCPDTBT and PCPDTBT:F4TCNQ hole acceptors. From the ratio of the PL emission intensity for perovskite without and with hole acceptor, the lower value for quenching can be estimated as 30 . Much deeper insight on the quenching efficiency can be obtained from PL kinetic measurements. Fig. 4b shows photoluminescence kinetics at $780 \mathrm{~nm}$ for the perovskite sample without quencher, with PCPDTBT and PCPDTBT:F4TCNQ. PL lifetime for perovskite is $160 \pm 20$ ns. Dramatic quenching, factor of 20000 (from $160 \mathrm{~ns}$ to $8 \mathrm{ps}$ ), was observed with hole acceptor PCPDTBT. Even stronger PL quenching was observed for perovskite/PCPDTBT:F4TCNQ. The PL lifetime was $\ll<5 \mathrm{ps,}$ i.e. below the limit of our instrument temporal response; therefore we can give only the lower limit of quenching: factor of 30000 (from $160 \mathrm{~ns}$ to $5 \mathrm{ps}$ ). Our obtained values for the lifetimes in the presence of pristine or F4TCNQ doped PCPDTBT are in agreement with reported values obtained with other quenchers in the range of $<1-40 \mathrm{ps}^{45-48} \mathrm{PL}$ measurements strongly indicate that both PCPDTBT and PCPDTBT:F4TCNQ can effectively transfer holes from the perovskite absorber. Therefore, we can conclude that the higher photovoltaic parameters obtained (mainly the $J_{\mathrm{sc}}$ ) for F4TCNQ doped PCPDTBT based devices should be largely attributed to a more efficient charge collection strongly correlated with the high conductivity.

To gain more insight into the charge recombination process at $\mathrm{TiO}_{2} /$ perovskite/HTM interfaces, electrochemical impedance spectroscopy (EIS) measurements were carried out for the PSC devices with and without doping F4TCNQ as HTMs. Fig. 5a and b present the Nyquist plots recorded under dark conditions with varied bias voltage in the frequency range from $10^{6}$ to $0.1 \mathrm{~Hz}$. According to the equivalent circuit model as reported previously, ${ }^{49}$ the main arc is supposed to be a combination of the recombination resistance $\left(R_{\text {rec }}\right)$ and the chemical capacitance of the film. By fitting the Nyquist plots, the relationship between the $R_{\text {rec }}$ and the bias voltage is obtained, as presented in Fig. 5c. It can be seen that the device with F4TCNQ doped PCPDTBT shows a larger $R_{\text {rec }}$ as compared to the pristine one at the same bias voltage, which may account for the slightly higher $V_{\text {oc }}$ obtained from the $J-V$ measurements.

Finally, the stability of PSCs devices based on F4TCNQ doped PCPDTBT, which were stored without encapsulation at ambient condition with humidity at $\sim 30 \%$ in the dark, was investigated for 840 hours. Fig. 6 displays the changes of photovoltaic parameters for a representative PSC device. The device showed an excellent long-term stability and remained over $85 \%$ of its initial PCE during the aging time as shown in Fig. 6b. The water contact angle of F4TCNQ doped PCPDTBT film is measured to be $99.49^{\circ}$ (Fig. 6c). The hydrophobic nature of F4TCNQ doped PCPDTBT can effectively prevent the water penetration into the perovskite layer, thus resulting in a good long-term stability. For comparison purpose, the stability of the well-known spiroOMeTAD was also studied in combination with F4TCNQ or LiTFSI and TBP as dopants. The devices were stored without
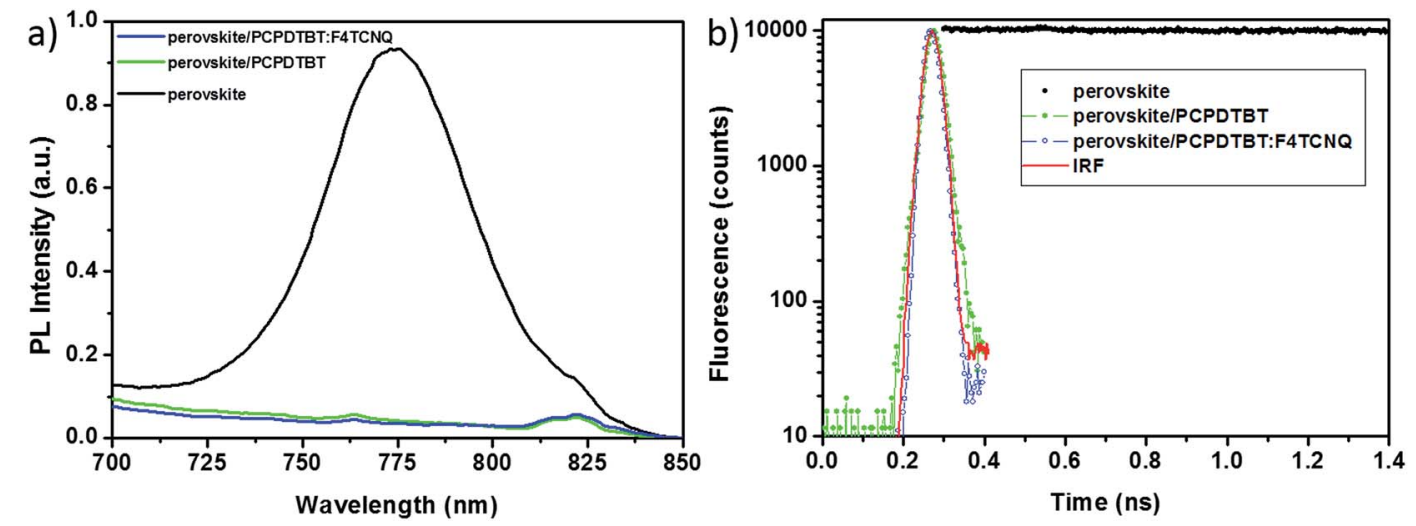

Fig. 4 (a) Steady-state PL of perovskite, perovskite with PCPDTBT and perovskite with F4TCNQ:PCPDTBT (6\% w/w) at excitation wavelength $600 \mathrm{~nm}$. (b) PL kinetics measured with TCSPC technique at $780 \mathrm{~nm}$ after excitation with $425 \mathrm{~nm}$. PL lifetime for perovskite is $160 \pm 20 \mathrm{~ns}$, for perovskite/PCPDTBT $=8$ ps and for perovskite/PCPDTBT:F4TCNQ $<<5$ ps. 

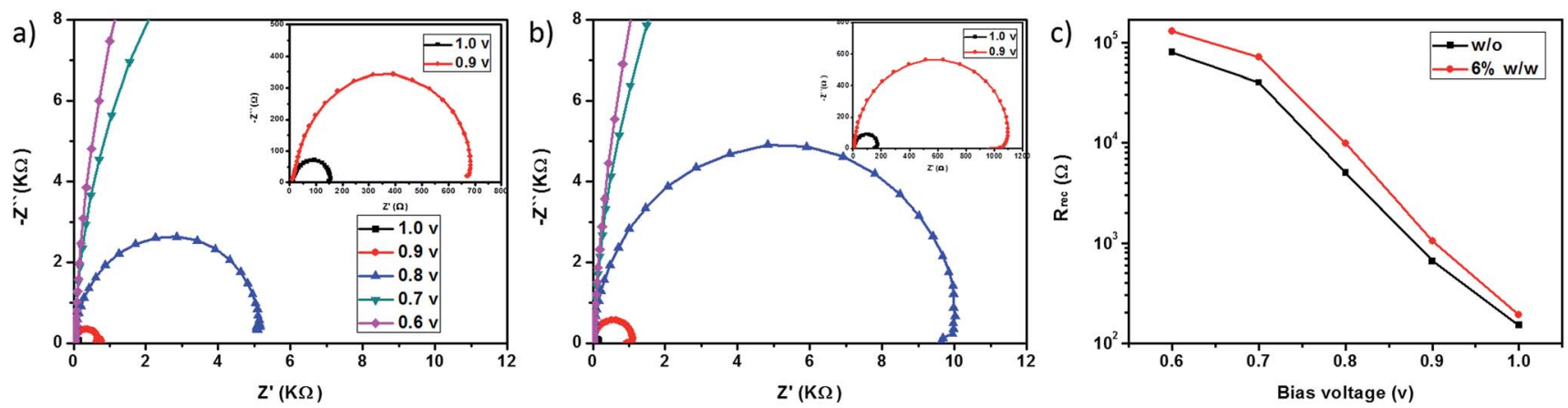

Fig. 5 Nyquist plots of the devices with (a) pristine PCPDTBT and (b) F4TCNQ doped PCPDTBT as HTMs measured in the dark over different forward biases (inset: enlarged high frequency region). (c) Plot of the recombination resistance $\left(R_{\text {rec }}\right)$ vs. bias voltages for the devices based on different HTMS.
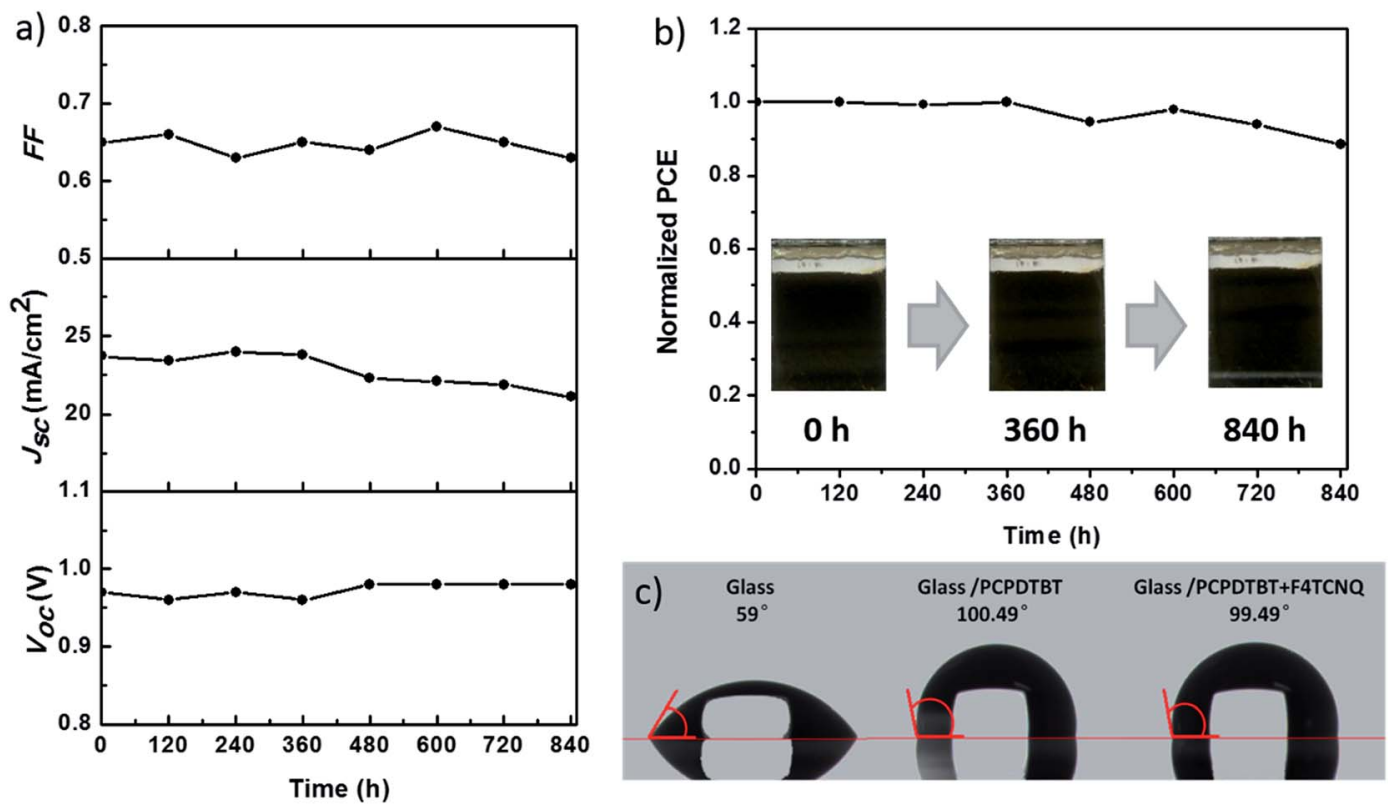

Fig. 6 (a) Change of photovoltaic parameters of $V_{\mathrm{OC}}, J_{\mathrm{sc}}$ and FF. (b) PCE variations based on PSCs with PCPDTBT:F4TCNQ composite as HTMs measured under AM 1.5 simulated sunlight ( $100 \mathrm{~mW} \mathrm{~cm}^{-2}$ irradiance) (inset shows the images of tested PSC device changing during this period of time). (c) Water contact angles of pristine and F4TCNQ doped PCPDTBT thin films deposited on the glass substrates.

encapsulation at ambient condition with humidity at $\sim 30 \%$ in the dark for 480 hours. The results are presented in Fig. S5 in the ESI. $\uparrow$ F4TCNQ doped spiro-OMeTAD exhibited a good longterm stability during the testing period, remaining $93 \%$ of its initial efficiency. By stark contrast, the efficiency of the device with LiTFSI and TBP maintained only $77 \%$ of its initial efficiency after 480 hours. Here, it emphasizes again that F4TCNQ is a stable dopant that can be further explored in other systems.

\section{Conclusions}

We have successfully demonstrated molecularly p-doping of D-A co-polymer PCPDTBT as an efficient HTM in PSCs. The bulk conductivity was enhanced by 4 orders of magnitude at an optimal doping condition. UV-vis and FTIR measurements confirmed that $\mathrm{p}$-doping occurs via the electron transfer from the HOMO level of PCPDTBT to the LUMO level of F4TCNQ, which gave rise to substantially higher bulk conductivity. The high conductivity led to an impressive overall efficiency of $15.1 \%$, largely owing to the significant enhancement of the photocurrent density strongly correlated with a more efficient charge collection. This is the highest reported value so far for PCPDTBT-based PSCs. The present work provides a general strategy for further optimizing a wide range of $\mathrm{D}-\mathrm{A}$ and other type of polymeric HTMs for highly efficient PSCs in the future.

\section{Acknowledgements}

This work was supported by the National Natural Science Foundation of China (21606039, 51661135021, 91233201), the Swedish Energy Agency, as well as the Knut and Alice Wallenberg Foundation. 


\section{References}

1 Z. Yu, F. Li and L. Sun, Energy Environ. Sci., 2015, 8, 760-775. 2 M. M. Lee, J. Teuscher, T. Miyasaka, T. N. Murakami and H. J. Snaith, Science, 2012, 338, 643-647.

3 H.-S. Kim, C.-R. Lee, J.-H. Im, K.-B. Lee, T. Moehl, A. Marchioro, S.-J. Moon, R. Humphry-Baker, J.-H. Yum, J. E. Moser, M. Grätzel and N.-G. Park, Sci. Rep., 2012, 2, 591-597.

4 W. Chen, Y. Wu, Y. Yue, J. Liu, W. Zhang, X. Yang, H. Chen, E. Bi, I. Ashraful, M. Grätzel and L. Han, Science, 2015, 350, 944-948.

5 F. Bella, G. Griffini, J.-P. Correa-Baena, G. Saracco, M. Grätzel, A. Hagfeldt, S. Turri and C. Gerbaldi, Science, 2016, 354, 203-206.

6 http://www.nrel.gov/ncpv/images/efficiency_chart.jpg.

7 Z. Yu and L. Sun, Adv. Energy Mater., 2015, 5, 1500213.

8 S. Ameen, M. A. Rub, S. A. Kosa, K. A. Alamry, M. S. Akhtar, H.-S. Shin, H.-K. Seo, A. M. Asiri and M. K. Nazeeruddin, ChemSusChem, 2016, 9, 10-27.

9 M. Saliba, T. Matsui, K. Domanski, J.-Y. Seo, A. Ummadisingu, S. M. Zakeeruddin, J.-P. Correa-Baena, W. R. Tress, A. Abate, A. Hagfeldt and M. Grätzel, Science, 2016, 354, 206-209.

10 B. Xu, D. Bi, Y. Hua, P. Liu, M. Cheng, M. Grätzel, L. Kloo, A. Hagfeldt and L. Sun, Energy Environ. Sci., 2016, 9, 873-877. 11 J. A. Christians, R. C. M. Fung and P. V. Kamat, J. Am. Chem. Soc., 2013, 136, 758-764.

12 P. Qin, S. Tanaka, S. Ito, N. Tetreault, K. Manabe, H. Nishino, M. K. Nazeeruddin and M. Grätzel, Nat. Commun., 2014, 5, 3834.

13 G. A. Sepalage, S. Meyer, A. Pascoe, A. D. Scully, F. Huang, U. Bach, Y.-B. Cheng and L. Spiccia, Adv. Funct. Mater., 2015, 25, 5650-5661.

14 N. J. Jeon, J. H. Noh, Y. C. Kim, W. S. Yang, S. Ryu and S. I. Seok, Nat. Mater., 2014, 13, 897-903.

15 N. J. Jeon, J. H. Noh, W. S. Yang, Y. C. Kim, S. Ryu, J. Seo and S. I. Seok, Nature, 2015, 517, 476-480.

16 Z. Zhu, Y. Bai, H. K. H. Lee, C. Mu, T. Zhang, L. Zhang, J. Wang, H. Yan, S. K. So and S. Yang, Adv. Funct. Mater., 2014, 24, 7357-7365.

17 S. Ameen, M. S. Akhtar, H.-K. Seo and H.-S. Shin, Langmuir, 2014, 30, 12786-12794.

18 J. Xiao, J. Shi, H. Liu, Y. Xu, S. Lv, Y. Luo, D. Li, Q. Meng and Y. Li, Adv. Energy Mater., 2015, 5, 1401943.

19 D. Bi, L. Yang, G. Boschloo, A. Hagfeldt and E. M. J. Johansson, J. Phys. Chem. Lett., 2013, 4, 1532-1536. 20 J. H. Heo, S. H. Im, J. H. Noh, T. N. Mandal, C.-S. Lim, J. A. Chang, Y. H. Lee, H.-j. Kim, A. Sarkar, K. NazeeruddinMd, M. Grätzel and S. I. Seok, Nat. Photonics, 2013, 7, 486-491.

21 Y. S. Kwon, J. Lim, H.-J. Yun, Y.-H. Kim and T. Park, Energy Environ. Sci., 2014, 7, 1454-1460.

22 W. S. Yang, J. H. Noh, N. J. Jeon, Y. C. Kim, S. Ryu, J. Seo and S. I. Seok, Science, 2015, 348, 1234-1237.
23 G.-W. Kim, G. Kang, J. Kim, G.-Y. Lee, H. I. Kim, L. Pyeon, J. Lee and T. Park, Energy Environ. Sci., 2016, 9, 2326-2333.

24 H.-C. Liao, T. L. D. Tam, P. Guo, Y. Wu, E. F. Manley, W. Huang, N. Zhou, C. M. M. Soe, B. Wang, M. R. Wasielewski, L. X. Chen, M. G. Kanatzidis, A. Facchetti, R. P. H. Chang and T. J. Marks, Adv. Energy Mater., 2016, 6, 1600502.

25 J. Peet, J. Y. Kim, N. E. Coates, W. L. Ma, D. Moses, A. J. Heeger and G. C. Bazan, Nat. Mater., 2007, 6, 497-500.

26 J. Kettle, M. Horie, L. A. Majewski, B. R. Saunders, S. Tuladhar, J. Nelson and M. L. Turner, Sol. Energy Mater. Sol. Cells, 2011, 95, 2186-2193.

27 P. C. Y. Chow, S. Gélinas, A. Rao and R. H. Friend, J. Am. Chem. Soc., 2014, 136, 3424-3429.

28 J. M. Marin-Beloqui, J. P. Hernandez and E. Palomares, Chem. Commun., 2014, 50, 14566-14569.

29 Q. Lin, A. Armin, R. C. R. Nagiri, P. L. Burn and P. Meredith, Nat. Photonics, 2015, 9, 106-112.

30 K.-H. Yim, G. L. Whiting, C. E. Murphy, J. J. M. Halls, J. H. Burroughes, R. H. Friend and J.-S. Kim, Adv. Mater., 2008, 20, 3319-3324.

31 D. Di Nuzzo, C. Fontanesi, R. Jones, S. Allard, I. Dumsch, U. Scherf, E. von Hauff, S. Schumacher and E. Da Como, Nat. Commun., 2015, 6, 6460.

32 Q. Wang, C. Bi and J. Huang, Nano Energy, 2015, 15, 275-280. 33 C. Bi, Q. Wang, Y. Shao, Y. Yuan, Z. Xiao and J. Huang, Nat. Commun., 2015, 6, 7747.

34 D. Liu, Y. Li, J. Yuan, Q. Hong, G. Shi, D. Yuan, J. Wei, C. Huang, J. Tang and M.-K. Fung, J. Mater. Chem. A, 2017, 5, 5701-5708.

35 Y. Zhang, M. Elawad, Z. Yu, X. Jiang, J. Lai and L. Sun, RSC Adv., 2016, 6, 108888-108895.

36 D. Bi, W. Tress, M. I. Dar, P. Gao, J. Luo, C. Renevier, K. Schenk, A. Abate, F. Giordano, J.-P. Correa Baena, J.-D. Decoppet, S. M. Zakeeruddin, M. K. Nazeeruddin, M. Grätzel and A. Hagfeldt, Sci. Adv., 2016, 2, e1501170.

37 X. Jiang, Z. Yu, J. Lai, Y. Zhang, N. Lei, D. Wang and L. Sun, Sci. China: Chem., 2017, 60, 423-430.

38 T. Leijtens, I. K. Ding, T. Giovenzana, J. T. Bloking, M. D. McGehee and A. Sellinger, ACS Nano, 2012, 6, 14551462.

39 H.-S. Kim and N.-G. Park, J. Phys. Chem. Lett., 2014, 5, 29272934.

40 R. Ihly, A.-M. Dowgiallo, M. Yang, P. Schulz, N. J. Stanton, O. G. Reid, A. J. Ferguson, K. Zhu, J. J. Berry and J. L. Blackburn, Energy Environ. Sci., 2016, 9, 1439-1449.

41 Z. Li, J. Tinkham, P. Schulz, M. Yang, D. H. Kim, J. Berry, A. Sellinger and K. Zhu, Adv. Energy Mater., 2017, 7, 1601451. 42 J. Xu, O. Voznyy, R. Comin, X. Gong, G. Walters, M. Liu, P. Kanjanaboos, X. Lan and E. H. Sargent, Adv. Mater., 2016, 28, 2807-2815.

43 S. N. Habisreutinger, T. Leijtens, G. E. Eperon, S. D. Stranks, R. J. Nicholas and H. J. Snaith, Nano Lett., 2014, 14, 55615568.

44 J. Zhang, B. Xu, M. B. Johansson, N. Vlachopoulos, G. Boschloo, L. Sun, E. M. J. Johansson and A. Hagfeldt, ACS Nano, 2016, 10, 6816-6825. 
45 N. Mondal and A. Samanta, Nanoscale, 2017, 9, 1878-1885. 46 S. Masi, S. Colella, A. Listorti, V. Roiati, A. Liscio, V. Palermo, A. Rizzo and G. Gigli, Sci. Rep., 2015, 5, 7725.

47 P. Maity, J. Dana and H. N. Ghosh, J. Phys. Chem. C, 2016, 120, 18348-18354.
48 H. He, Q. Yu, H. Li, J. Li, J. Si, Y. Jin, N. Wang, J. Wang, J. He, X. Wang, Y. Zhang and Z. Ye, Nat. Commun., 2016, 7, 10896. 49 S. Lv, L. Han, J. Xiao, L. Zhu, J. Shi, H. Wei, Y. Xu, J. Dong, X. Xu, D. Li, S. Wang, Y. Luo, Q. Meng and X. Li, Chem. Commun., 2014, 50, 6931-6934. 